\title{
MicroRNA-10b mediates TGF-ß1-regulated glioblastoma proliferation, migration and epithelial-mesenchymal transition
}

\author{
CHENGYUAN MA $^{1 *}$, FENG WEI ${ }^{3 *}$, HUAN XIA $^{2}$, HAIYU LIU $^{1}$, XUECHAO DONG $^{4}$,

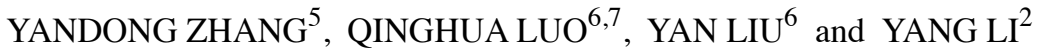 \\ Departments of ${ }^{1}$ Neurosurgery, ${ }^{2}$ Respiratory Medicine, ${ }^{3}$ Hepatobiliary and Pancreas Surgery, \\ ${ }^{4}$ Intensive Care Unit, and ${ }^{5}$ Rheumatology, The First Hospital of Jilin University, Changchun, Jilin 130021; \\ ${ }^{6}$ Genetic Engineering Laboratory of PLA, The Eleventh Institute of Academy of Military Medical Sciences of PLA, \\ Changchun, Jilin 130122; ${ }^{7}$ College of Veterinary Meficine, Yangzhou University, Yangzhou, Jiangsu 225009, P.R. China
}

Received January 18, 2017; Accepted March 20, 2017

DOI: $10.3892 /$ ijo.2017.3947

\begin{abstract}
Although it is well known that exaggerated proliferation, metastasis and the mesenchymal subtype is related with worst prognoses in glioblastoma (GBM) and that transforming growth factor- $\beta 1$ (TGF- $\beta 1$ ) is a potent factor in regulating the proliferation, migration and epithelial-mesenchymal transition (EMT) phenotype of GBM, the detailed mechanisms are still far from elucidated. MicroRNAs (miRNAs) are small noncoding RNAs which play critical roles in various diseases by regulating target gene expression. We report that miR-10b, a molecule downstream of TGF- $\beta 1$, is involved in TGF- $\beta 1$ regulated GBM cell proliferation, migration and EMT. We found that exposure of GBM cells to TGF- $\beta 1$ significantly upregulated miR-10b expression. Overexpression of miR-10b promotes GBM cell proliferation, migration and EMT, whereas depletion of miR-10b obtained reverse effects. Further studies uncovered that some tumor-associated genes including epithelial cadherin (E-cadherin), apoptotic protease activating factor 1 (Apaf-1) and phosphatase and tensin homolog (PTEN) are target genes of miR-10b. In human GBM xenografts, antagomiR directed against miR-10b markedly suppressed tumor growth, and the tumor volume shrunk from $1252.5 \pm 285$ to $873.4 \pm 205 \mathrm{~mm}^{3}$ after antagomiR-10b treatment for 3 weeks compared with the control group $(\mathrm{P}<0.01)$. Taken together, our data collectively demonstrate that the proliferation, migration and EMT features of GBM cells can be regulated by TGF- $\beta 1$
\end{abstract}

Correspondence to: Professor Yang Li, Department of Respiratory Medicine, The First Hospital of Jilin University, No. 71 Xinmin Road, Changchun, Jilin 130021, P.R. China

E-mail: pangya-lee@163.com

Professor Yan Liu, Genetic Engineering Laboratory, The Eleventh Institute of Academy of Military Medical Sciences of PLA, No. 666 Liuying West Road, Changchun, Jilin 130122, P.R. China E-mail: liu820512@163.com

*Contributed equally

Key words: glioblastoma, proliferation, migration, epithelialmesenchymal transition, microRNA-10b, TGF- $\beta$ stimulation through controlling miR-10b. Thus, our findings provide a rationale for targeting TGF- $\beta 1$ or miR-10b for the treatment of GBM.

\section{Introduction}

Glioblastoma multiforme (GBM), a highly malignant grade 4 glioma, is the most common primary cancer of the brain. Surgery combined with radiotherapy and chemotherapy is still the standard treatment for GBM patients (1). However, the high mobility and strong invasive properties of GBM result in a high inevitable recurrence rate and a poor median survival of 14.6 months for patients (2). Thus, there is a pressing need to reveal the molecular mechanisms of GBM invasion for novel therapeutic avenue development.

Epithelial-mesenchymal transition (EMT) is a process in which epithelial cells lose their polarity and adhesion ability and become mesenchymal stem cells gaining migratory and invasive properties (3). Accumulating evidence has showed that EMT also occurs and plays a critical role in the initiation of metastasis for tumor progression. It is well known that transforming growth factor- $\beta 1$ (TGF- $\beta 1$ ) signaling pathway plays a principle role in accelerating epithelial plasticity that may progress to EMT (4). However, TGF- $\beta 1$-induced EMT does not happen in some non-invasive tumor cells in vitro (5). On top of that, TGF- $\beta 1$ has a dual role and it can act as either a tumor suppressor or promoter depending on the stages and types of the tumor $(3,4)$. Thus, the detailed mechanism that TGF- $\beta 1$ regulates GBM has not been fully explored to date.

MicroRNAs (miRNAs) are small non-coding RNAs that post-transcriptionally repress target gene expression by inhibiting translation or promoting mRNA degradation (6). Approximately $35828 \mathrm{miRNAs}$ have been found to be expressed in 223 species (http://microrna.sanger.ac.uk), which facilitates the possibility of reciprocal interactions between miRNAs and finely regulating gene expression by miRNAs. The most compelling evidence is that the aberrant production of miRNAs has been widely recognized as a main character of various human diseases, including developmental abnormalities, autoimmune diseases and cancer (7-9). Mounting evidence indicated that miRNAs play pivotal roles in 
$\operatorname{GBM}(10,11)$. For example, miR-146b-5p is downregulated and triggers the miR-146b-5p/Hu antigen R (HuR)/lincRNA-p21/ $\beta$-catenin signaling pathway in glioma stem cells (GSCs) and miR-146b-5p overexpression attenuates stemness and radioresistance of GSCs (12).

miR-10b has been reported to be an oncogenic miRNA which can regulate growth and metastasis of various types of cancer (13-15). By using pleiotropic mechanisms, miR-10b widely participated in the regulating of cancer cell proliferation, migration, invasion and EMT. Although several target genes of miR-10b have been designated in GBM and other tumors, their regulation looks cell- and context-specific $(13,16,17)$. Thus, further studies are still needed to uncover the detailed mechanisms underlying miR-10b functions in regulating GBM progression.

In the present study, we investigated the role of miR-10b in TGF- $\beta 1$-mediated GBM proliferation, migration and EMT. We found that miR-10b is apparently upregulated by TGF- $\beta 1$ in U251 and U87 cells. Further studies uncovered that TGF- $\beta 1$ remarkably promoted GBM cell proliferation, migration and EMT. All these effects were achieved through regulating miR-10b as miR-10b mimics promoted, whereas miR-10b inhibitor reversed, the effects of TGF- $\beta 1$. In addition, several proliferation-, invasion- and EMT-associated genes including epithelial cadherin (E-cadherin), apoptotic protease activating factor 1 (Apaf-1) and phosphatase and tensin homolog (PTEN) are the targets of miR-10b. When xenograft models were used to investigate the miR-10b potency as therapeutic target in vivo, results showed that antagomiR-10b apparently suppressed tumor progression. In summary, our data collectively demonstrated that miR-10b can be used as a potential therapeutic target for the treatment of GBM.

\section{Materials and methods}

Clinical specimens. Glioblastoma tissues $(\mathrm{n}=15)$ were obtained from the First Hospital of Jilin University (Changchun, China). All of the procedures involving specimens obtained from human subjects were performed under protocols approved by the Jilin University Ethic Committee. Written informed consent was also obtained from all subjects before the study. None of the patients received radiation therapy or chemotherapy before surgical resection. All tissue samples were snap-frozen in liquid nitrogen and stored at $-70^{\circ} \mathrm{C}$ until use.

Cell lines and cell culture. Human glioma cell lines U87 and U251 were purchased from the China Academia Sinica Cell Repository (Shanghai, China). The cells were cultured in Dulbecco's modified Eagle' medium (DMEM) supplemented with $10 \%$ fetal bovine serum (FBS), $50 \mathrm{U} / \mathrm{ml}$ penicillin/streptomycin and $2 \mathrm{mM} \mathrm{L-glutamine} \mathrm{(Gibco,} \mathrm{Carlsbad,} \mathrm{CA,} \mathrm{USA).}$ All the cell lines were incubated at $37^{\circ} \mathrm{C}$ in a $\mathrm{CO}_{2}$ incubator. For cell treatment, $5 \mathrm{ng} / \mathrm{ml}$ of TGF- $\beta$ was added or $100 \mathrm{nM}$ miR-10b mimics or inhibitor was transfected into the cells with Lipofectamine 3000 as indicated. All the reagents were purchased from Life Technologies (Grand Island, NY, USA).

RNA isolation and real-time PCR. Total RNA of GBM cells was isolated with TRIzol, and cDNA was then generated using the reverse transcription kits (TaqMan ${ }^{\circledR}$ MicroRNA Reverse
Transcription kit for miRNA, PrimeScript ${ }^{\circledR}$ First Strand cDNA synthesis kit for general genes) following the protocols of the manufacturer (all from Life Technologies, Grand Island, NY). Aliquots of the reaction products were then used for real-time PCR with an ABI PRISM 7500 Fast system using the following parameters: initial denaturation at $95^{\circ} \mathrm{C}$ for $10 \mathrm{~min}$, followed by 40 cycles of $95^{\circ} \mathrm{C}$ for $15 \mathrm{sec}, 60^{\circ} \mathrm{C}$ for $1 \mathrm{~min}$ and $72^{\circ} \mathrm{C}$ for $45 \mathrm{sec}$. The expression of miR-10b was normalized to U6B miRNA and E-cadherin, Apaf-1 and PTEN were normalized to GAPDH mRNA. All PCR experiments were performed in triplicate.

Western blotassay. U251 cells were thoroughly lysed in ice-cold RIPA buffer (P0013C; Beyotime Institute of Biotechnology, Haimen, China) for $45 \mathrm{~min}$. Then, $\sim 40 \mu \mathrm{g}$ protein was subjected to SDS-PAGE and transferred to polyvinylidene difluoride (PVDF) membranes. After incubating in blocking buffer [phosphate-buffered saline (PBS) containing 3\% BSA] for $1 \mathrm{~h}$ at room temperature, the membranes were subsequently incubated with E-cadherin (1:3,000; Abcam, Cambridge, MA, USA), vimentin (1:3,000; Abcam), Apaf-1 (1:3,000; BD Biosciences, San Diego, CA), PTEN (1:600; Abcam), Tubulin (1:1,000; CWBio, Beijing, China) or $\beta$-actin $(1: 4,000 ;$ CWBio $)$ monoclonal antibodies and HRP-conjugated secondary antibodies, and the specific immunoreactive proteins were visualized through enhanced chemiluminescence.

Vector construction and luciferase reporter assays. The Dual-luciferase vectors were constructed by synthesizing the seed sequences in the 3'-UTRs of E-cadherin, Apaf-1 and PTEN, or the reverse complementary sequence of miR-10b (rcmiR-10b) and inserting the annealing products into the psiCHECK-2 vector. The corresponding mutant vectors were also constructed by introducing 3-bp mutations into the seed sequences. To verify the specific targeting of these genes by miR-10b, HEK293T cells were seeded in 24-well plates $\left(1.5 \times 10^{6} /\right.$ well $)$ and transfected with $0.8 \mu \mathrm{g}$ of the endotoxinfree recombinant vectors, either alone or in combination with 50-nM miR-10b precursors or inhibitors. Luciferase activities were measured $24 \mathrm{~h}$ later using the Dual-luciferase reporter assay system.

EdU proliferation assay. EdU (5-ethynyl-2'-deoxyuridine) proliferation assay was performed to measure cell proliferation. In brief, cells treated as indicated were seeded in 48 -well plates $\left(2 \times 10^{4}\right.$ cells/well) and cultured for $24 \mathrm{~h}$. Subsequently, the cells were incubated for $3 \mathrm{~h}$ in serum-free DMEM supplemented with $30 \mu \mathrm{M}$ EdU (Guangzhou RiboBio, Co., Ltd., Guangzhou, China) after being washed in PBS thrice. Afterwards the cells were fixed with $4 \%$ polyformaldehyde in PBS at room temperature for $30 \mathrm{~min}$. Finally, cells were incubated with Apollo staining solution and Hoechst 33342 for $30 \mathrm{~min}$ each. Proliferation index was presented as the percentage of EdU-positive cells relative to the total cell numbers. Images were acquired using a fluorescent microscope (Olympus IX73) and cells selected from five random fields were counted.

Wound closure assay. U251 and U87 cells were treated as indicated and a wound closure assay was performed to evaluate the cell migration ability. The cells were plated at $1.5 \times 10^{6}$ cells/ 

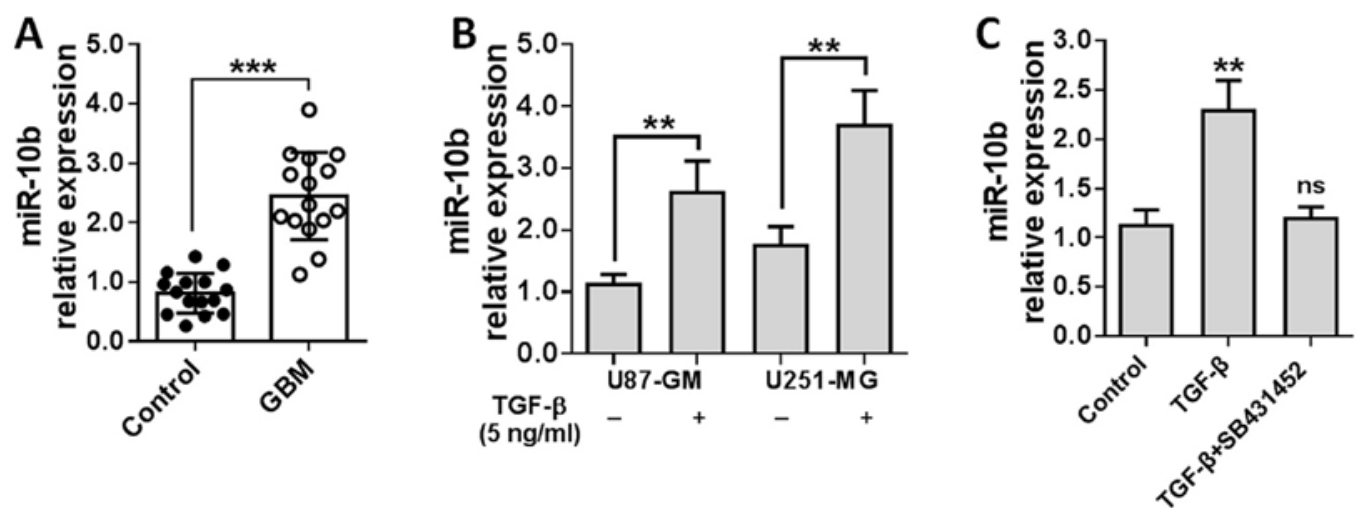

Figure 1. miR-10b is upregulated in GBM tissues or TGF- $\beta 1$-stimulated GBM cell lines. (A) Total RNA was isolated from tumor and adjacent non-tumor tissues from 15 GBM patients and miR-10a levels were detected by real-time PCR. (B) U87 and U251 cells were treated with TGF- $\beta 1$ for $24 \mathrm{~h}$ and miR-10b expression was measured by quantitative PCR. (C) U251 cells were treated with TGF- $\beta 1$ alone or in the presence of TGF- $\beta$ receptor inhibitor SB431452, and miR-10b was detected $24 \mathrm{~h}$ later. ${ }^{\mathrm{n}} \mathrm{P}>0.05,{ }^{* * *} \mathrm{P}<0.01,{ }^{* * * *} \mathrm{P}<0.001$ compared with the control group. Data are representative of three experiments.

$\mathrm{ml}$ in 12-well dishes and stayed in the incubator at $37^{\circ} \mathrm{C}$ until a confluent cell layer was established. A scratch in the cells was then made with a sterile pipette tip, and both the numbers and the average distance that cells moved from the edge of the scratch towards the center were measured $24 \mathrm{~h}$ later.

Nude mouse xenograft model. Animal experiments were careful performed following the guidelines of Jilin University Institutional Animal Care and Use Committee (IACUC) and were approved by the Institutional Animal Ethics Committee of Jilin University. U251 cells $\left(1 \times 10^{6}\right)$ transfected with antagmiR-10b, agomiR-10b or miR-scramble were resuspended in HBSS and injected subcutaneously into the flank region of female athymic (nu/nu) mice aged at 4-6 weeks (Beijing, China). The tumors were allowed to grow to average volume of $200 \mathrm{~mm}^{3}$ prior to initiation of treatment. PBS and the miR-scramble were used as negative controls. The tumor volume (V) was measured every other day with a slide caliper and calculated by the formula: $V=4 / 3 \times \pi$ [length $/ 2 \times$ (width $/ 2)^{2}$ ]. All mice were sacrificed after 18 days of treatment. Finally, the mice were sacrificed, and the tumors were isolated and snap-frozen in liquid nitrogen for following experiments.

Statistical analysis. All statistical analyses were performed using GraphPad Prism software. All data from at least three independent experiments were analyzed with the Student's t-test; $\mathrm{P}<0.05$ were considered statistically significant.

\section{Results}

miR-10b is upregulated in TGF- $\beta 1$-stimulated GBM cells. Considering that TGF- $\beta 1$ plays critical and paradoxical roles in GBM but the detailed mechanism is still far from elucidated, we wondered whether miRNAs play critical roles in TGF- $\beta 1$-treated GBM cells. U251 and U87 cells were treated with $5 \mathrm{ng} / \mathrm{ml}$ TGF- $\beta 1$ for $24 \mathrm{~h}$, and total RNAs were isolated from the cells and the miRNA expression profile was determined by miRNA microarray. Approximately 20 miRNAs were differentially expressed between the TGF- $\beta 1$-treated and control cells, among which miR-10b was significantly upregulated by TGF- $\beta 1$ in U251 and U87 cells.
To confirm this finding, miR-10b level was detected in the specimens collected from 15 GBM patients with real-time PCR. As shown in Fig. 1A, miR-10b was significantly overexpressed in GBM tissues relative to adjacent non-tumor tissues. Then, U251 and U87 cells were stimulated by TGF- $\beta 1$ for $24 \mathrm{~h}$ and miR-10b levels were measured. Results showed that miR-10b expression was elevated 2 -3-fold in TGF- $\beta 1$-treated GBM cells (Fig. 1B). To further determine whether miR-10b upregulation is TGF- $\beta 1$ specific, we treated U251 cells with TGF- $\beta 1$ in the presence of TGF- $\beta$ receptor inhibitor (SB431452). As shown in Fig. $1 \mathrm{C}$, blockade of TGF- $\beta 1$ signaling notably reversed the induction of miR-10b. All these data suggested that miR-10b is induced in GBM in a TGF- $\beta 1$-dependent manner.

miR-10b mediates TGF- $\beta 1$-induced GBM cell proliferation. Although several studies found that miR-10b is predominantly expressed in GBM but absent in normal brain tissues (18-20), whether miR-10b participate in TGF- $\beta 1$-mediated GBM cell proliferation, migration and EMT are not previously reported.

It is well known that the TGF- $\beta 1$ exerts both tumor promoting and tumor suppressive functions during cancer progression, in a variety of cancers, depending on the stages and types of the tumors. To investigate the effects of TGF- $\beta 1$ on GBM cells, U87 cells were treated with TGF- $\beta 1$ for $72 \mathrm{~h}$ and cells were stained with crystal violet. As shown in Fig. 2A, more plurinuclear cells were found in TGF- $\beta 1$-treated cells than untreated ones, which indicated that TGF- $\beta 1$-treated sample contains a reasonable number of proliferating cells under mitosis. Then, both U87 and U251 cells were treated with TGF- $\beta 1$ and cells were counted $72 \mathrm{~h}$ later. Results showed that TGF- $\beta 1$ exerted potent proliferation-stimulation effect on GBM cells (Fig. 2B and C, panel 2). As miR-10b was upregulated by TGF- $\beta 1$, we speculated that miR-10b mediated the effects of TGF- $\beta 1$ on U251 and U87 cells. To verify this hypothesis, U251 and U87 cells were treated with miR-10b mimics (miR-10b), miR-10b inhibitor (anti-miR-10b), either alone or in the combination with TGF- $\beta 1$. Results showed that miR-10b mimics (Fig. 2B and C, panel 3) promoted, whereas miR-10b inhibitor suppressed (Fig. 2B and C, panel 4) GBM cell proliferation. When treated in combination with TGF- $\beta 1$ 
A

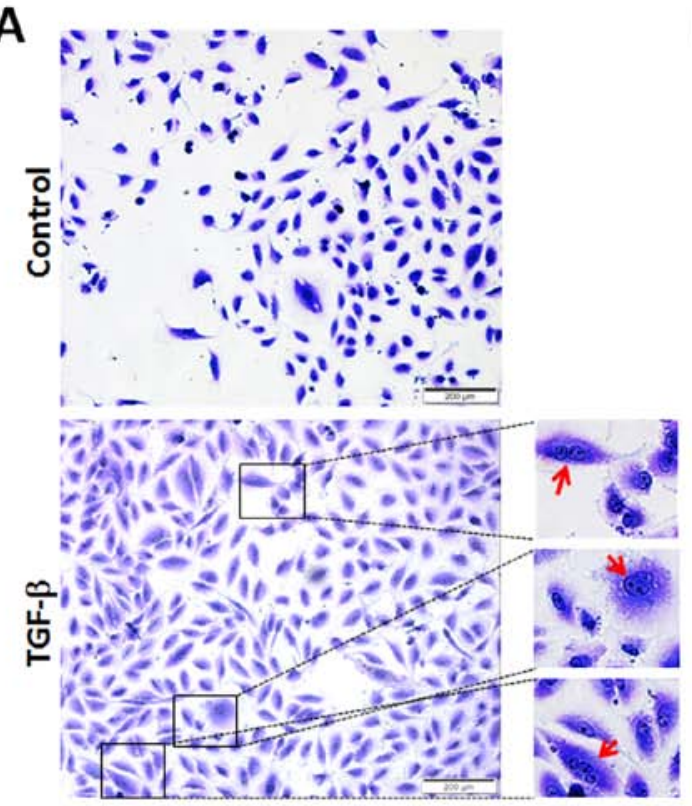

C

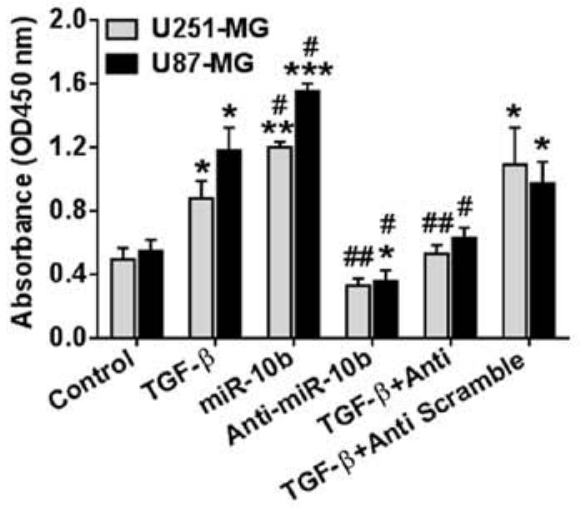

B
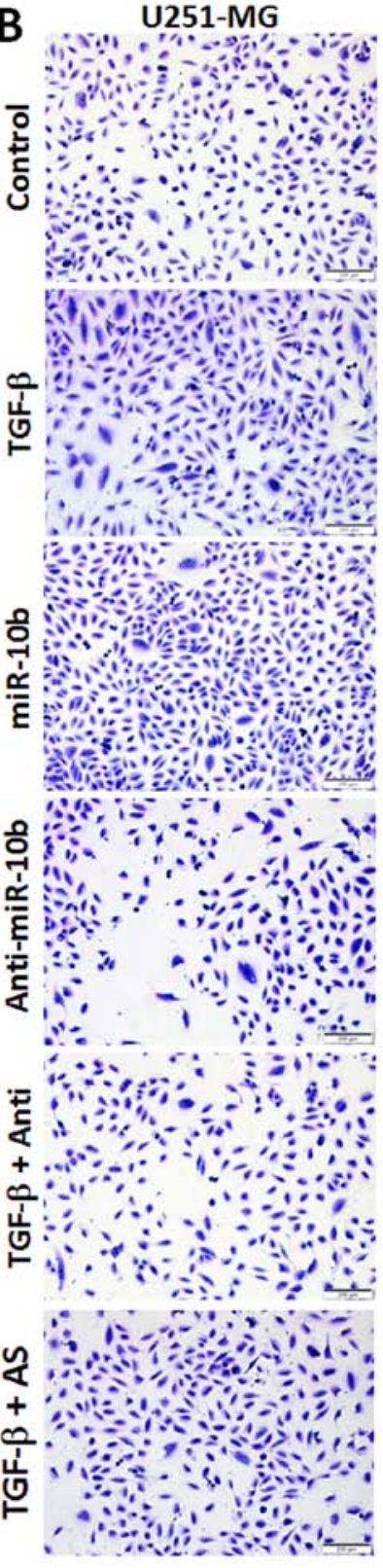

U87-MG

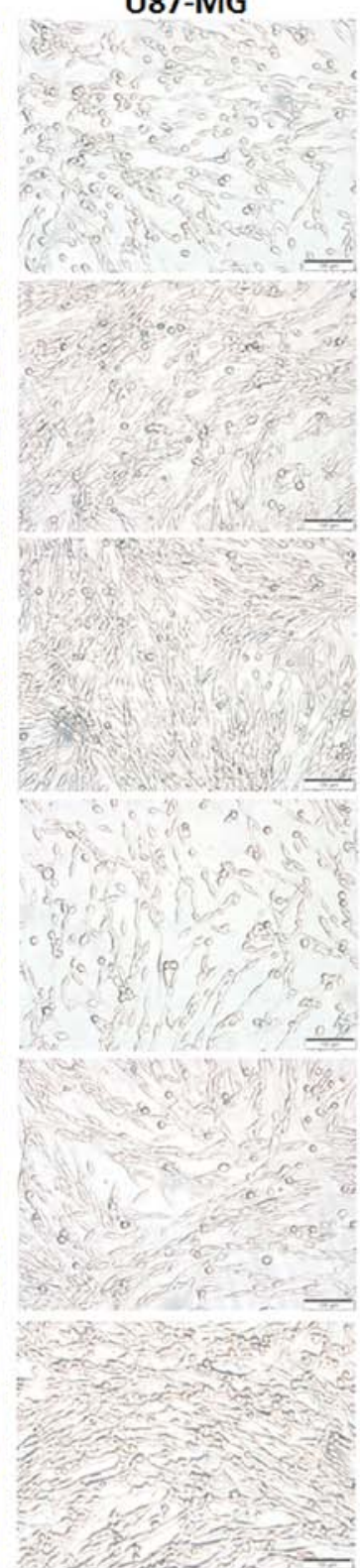

Figure 2. TGF- $\beta 1$ promotes GBM cell proliferation via upregulating miR-10b. (A) U251 cells were stimulated with recombinant TGF- $\beta 1$ (5 ng/ml) for $24 \mathrm{~h}$ and cells were stained with crystal violet according to standard protocols to observe cell growth. (B) Both U87 and U251 cells were treated as indicated for $24 \mathrm{~h}$, and cell numbers in 5 randomly selected fields were counted. (C) Statistical analysis data of (B). ${ }^{*} \mathrm{P}<0.05,{ }^{* *} \mathrm{P}<0.01,{ }^{* * * *} \mathrm{P}<0.001$ compared with the control group. ${ }^{~} \mathrm{P}<0.05,{ }^{\# \#} \mathrm{P}<0.01$ compared with TGF- $\beta 1$-treated group. Data are representative of three experiments. Bar, $200 \mu \mathrm{m}$. miR-10b, miR-10b precursor; anti-miR-10b/Anti, miR-10b inhibitor; AS, inhibitor scramble.

and anti-miR-10b, TGF- $\beta 1$-mediated cell proliferation was remarkably reversed (Fig. $2 \mathrm{~B}$ and $\mathrm{C}$, panel 5). This is a unique feature of the miR-10b inhibitor as the scramble inhibitor (Fig. 2B and C, panel 6) has no effect on TGF- $\beta 1$. All the data suggested that TGF- $\beta 1$ promotes GBM cell proliferation at least partially through regulating miR-10b.

To further confirm that TGF- $\beta 1$ directly stimulated GBM cell proliferation, we treated U251 and U87 cells with TGF- $\beta 1$ and determined the cell growth by EdU proliferation assays. Results showed that growth of the cells was apparently accelerated in the presence of TGF- $\beta 1$ (Fig. $3 \mathrm{~A}$ and B). In addition, the effect of TGF- $\beta 1$ was remarkably shielded in the presence of miR-10a inhibitors. All these data collectively indicated that miR-10b mediated TGF- $\beta 1$-induced GBM cell proliferation.
miR-10b enhances GBM cell migration. The migration, invasion and infiltration of tumor cells are one of the significant contributors to mortality in GBM patients. Although GBM cells can acquire enhanced invasive features following stimulation with some secretory cytokines like TGF- $\beta 1$ and which contributes to the heterogeneity of $\operatorname{GBM}(21,22)$, the detailed mechanism are still unclear. Several genes including ZEB1, Crk-like (CrkL) and HOXA13 have been reported to be associated with TGF- $\beta 1$-induced migration of GBM cells (22-24). To measure the roles that miR-10b may play in TGF- $\beta 1$-regulated GBM cell migration, the wound closure assay was performed and as expected, treatment with TGF- $\beta 1$ or miR-10b mimics significantly promoted the sound healing ability of both U87 (Fig. 4A and C) and U251 (Fig. 4B and D) cells, whereas the 
A

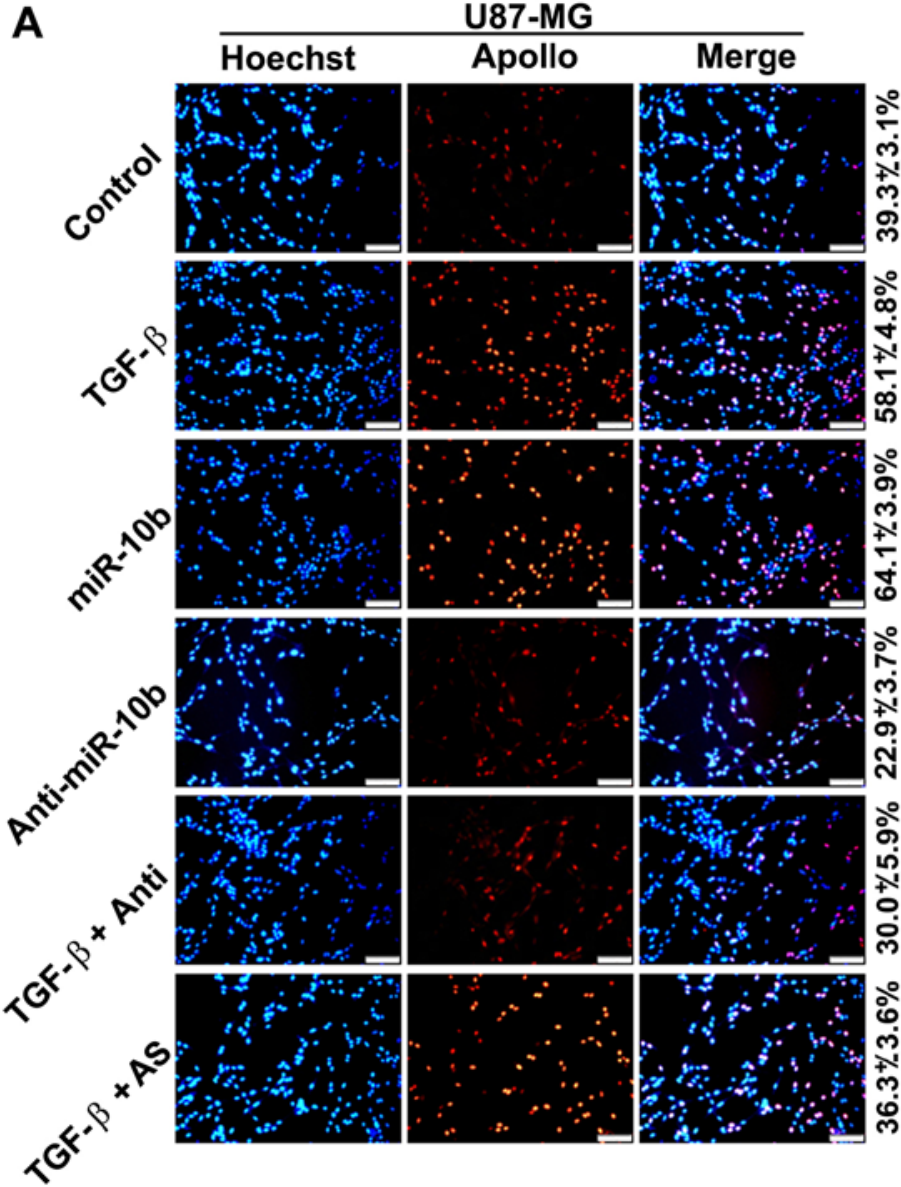

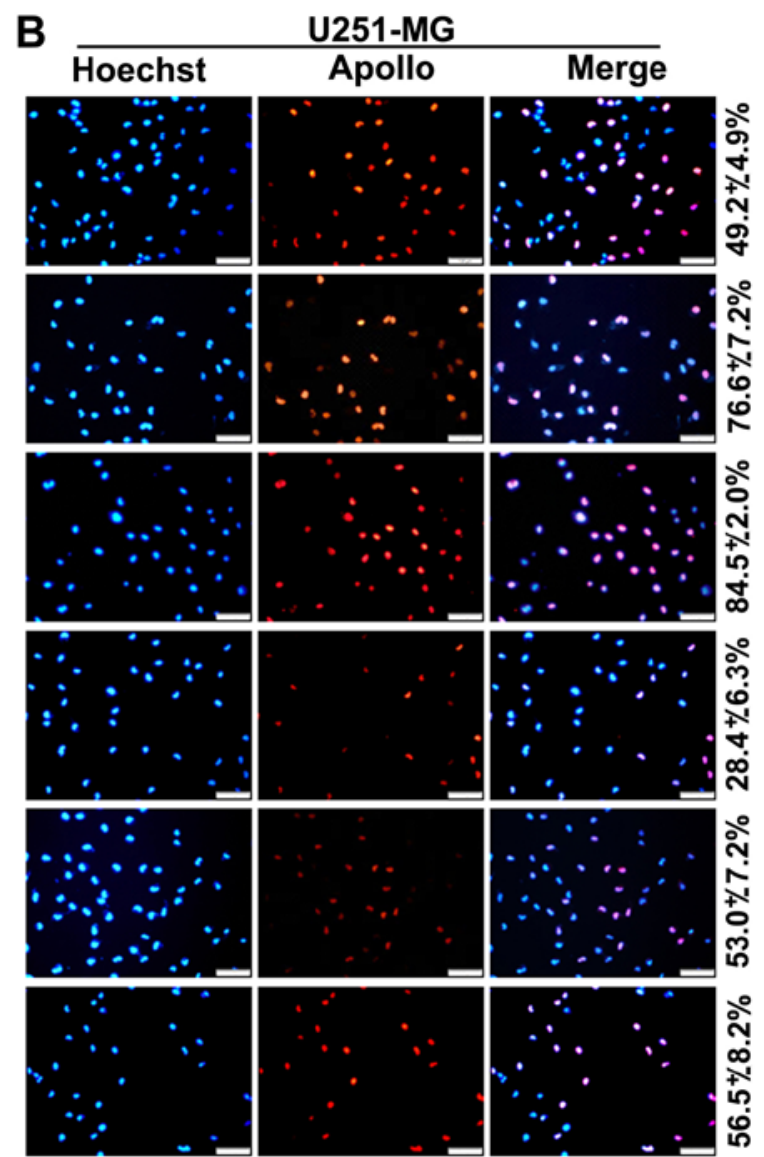

Figure 3. miR-10b mediates TGF- $\beta 1$-induced GBM cell proliferation. (A) U87 and (B) U251 cells were cultured for $72 \mathrm{~h}$ in the presence of TGF- $\beta 1$ (5 ng/ml) and subjected to EdU proliferation assays. Blue, Hoechst staining of nuclei in total cells. Red, Apollo staining of EdU in DNA of proliferating cells. The percentage of proliferating cells is given in the pictures, respectively. Bar, $100 \mu \mathrm{m}$. miR-10b, miR-10b precursor; anti-miR-10b/Anti, miR-10b inhibitor; AS, inhibitor scramble.

miR-10b inhibitor effectively weakened the effects of TGF- $\beta 1$. These data reveal that miR-10b is involved in the regulation of GBM cell migration.

miR-10b promotes EMT and lamellipodia formation in GBM cell lines. Considering EMT is a common feature of various tumors which may be closely associated with tumor invasion and metastasis and TGF- $\beta 1$ is one of the most potent inducers of EMT $(25,26)$. In addition, TGF- $\beta 1$ is also an important cytokine in the GBM microenvironment $(27,28)$. We investigated whether miR-10b participates in the EMT of GBM cells. As shown in Fig. 5A, treatment with TGF- $\beta 1$ (panel 2) or miR-10b mimics (panel 3) induced a remarkable morphological change of U87 and U251 cells from pebble shape to the long fusiform shape, reminiscent of EMT. However, miR-10b inhibitor makes the cells round and of cobblestone-appearance (panel 4). To further confirm whether miR-10b induce EMT in GBM cells, we measured the expression level of EMT-associated markers in U251 cells by western blot analysis. We found that the expression of E-cadherin, a classical epithelial marker, was apparently suppressed after miR-10b treatment, whereas vimentin, a mesenchymal marker, was increased significantly (Fig. 5B). Consistently, miR-10b inhibitor-transfected cells showed reverse properties. These data indicated that miR-10b is involved in TGF- $\beta 1$-induced EMT in GBM cells.
It is well known that both morphologic changes in EMT and migration and invasion of GBM cells require dynamic reorganization of the actin cytoskeleton including lamellipodial extensions, focal adhesions and stress fiber formation at the leading edge of GBM. We assessed whether miR-10b participates in the reorganization of the actin cytoskeleton. As shown in Fig. 5C, TGF- $\beta 1$ and miR-10b mimics induced significant lamellipodia formation in U87 and U251 cells. This is consistent with the aforementioned data that miR-10b possesses the property to mediate TGF- $\beta 1$-induced GBM cell migration and EMT.

miR-10b targets E-cadherin, caspase-9, Apaf-1 and PTEN. To investigate the pathological role of miR-10b in GBM, miR-10b target genes were predicted using microRNA online prediction software (http://www.microRNA.org). We focused on proliferation-, invasion- and EMT-related genes and E-cadherin, Apaf-1 and PTEN are screened as miR-10b candidate target genes for further validation. E-cadherin is encoded by the CDH1 gene which mediates cell-cell contact at the basolateral membrane and is a hallmark of epithelial cells. Loss of E-cadherin is a marker of EMT (29). Apaf-1 is a cofactor of caspase-9 and can regulate mitochondria-mediated apoptosis (30). PTEN is a specific tumor suppressor gene which can regulate GBM cell growth, apoptosis, adhesion, 


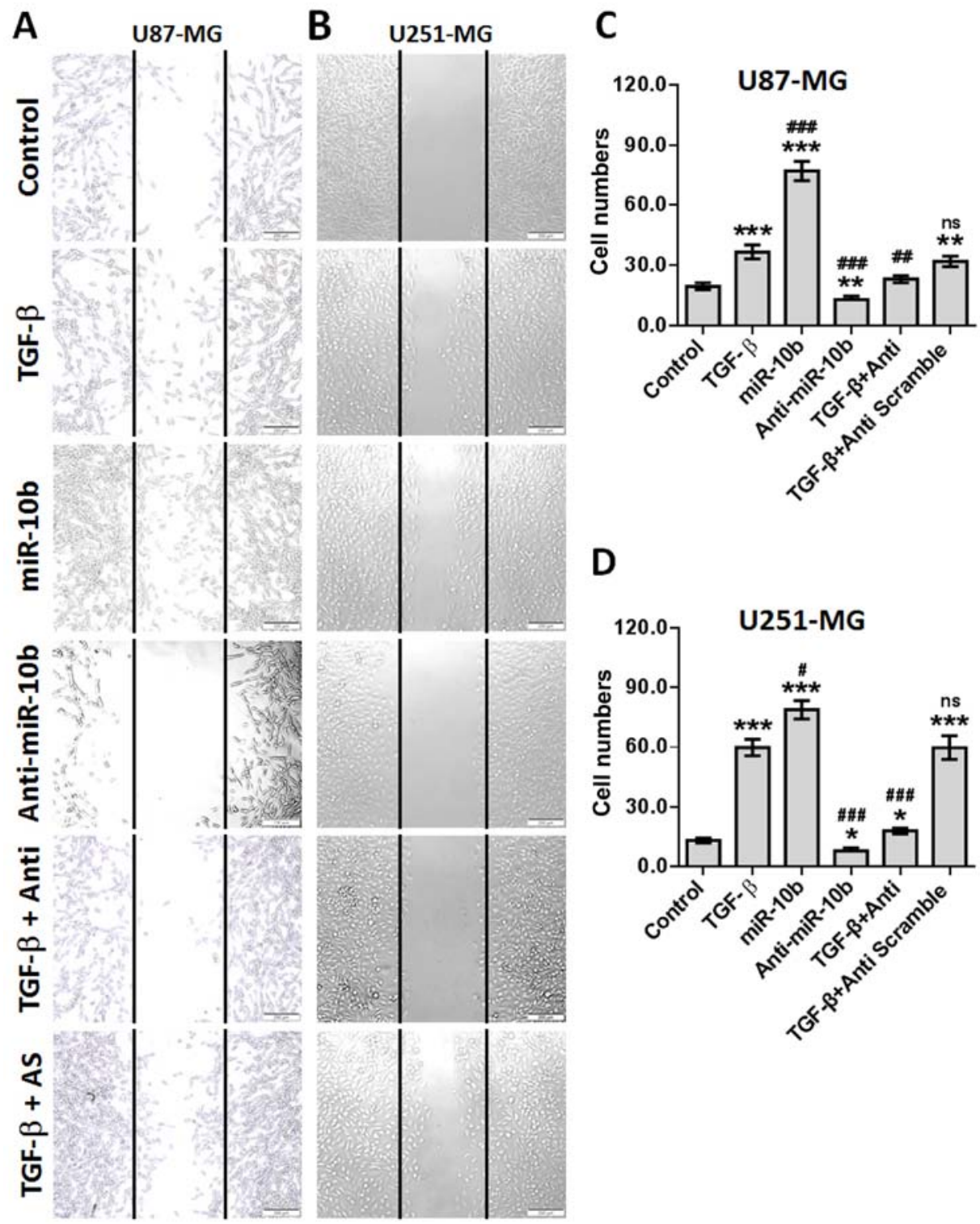

Figure 4. Promotion of GBM cell migration by overexpression of miR-10b. (A) U87 and (B) U251 cells were treated as indicated and then grew to confluence in 12-well dishes. The cultures were subjected to cell migration assay at $24 \mathrm{~h}$ after cell layer scratching. (C and D) The cell motility was evaluated by counting the numbers of the migrated cells along the margins of the scratch towards the denuded area. ${ }^{*} \mathrm{P}<0.05,{ }^{* * *} \mathrm{P}<0.01,{ }^{* * * *} \mathrm{P}<0.001$ compared with the control group. ${ }^{\#} \mathrm{P}<0.05,{ }^{\# \#} \mathrm{P}<0.01,{ }^{\# \#} \mathrm{P}<0.001$ compared with TGF- $\beta 1$-treated group. Data are representative of three independent experiments. Bar, $200 \mu \mathrm{m}$. miR-10b, miR-10b precursor; Anti-miR-10b/Anti, miR-10b inhibitor; AS, inhibitor scramble.

invasion and metastasis and has been used for GBM prognosis evaluation (31). All these genes are closely related with the proliferation, invasion and EMT of various tumor cells.

To determine whether these genes are real target genes of miR-10b in GBM cells, we constructed luciferase reporter vectors containing the theoretical seed sequences in the 3'-UTR of E-cadherin, Apaf-1 and PTEN as well as the corresponding mutant vectors. All these endo-free vectors were transfected into HEK293T cells, either alone or in combination with miR-10b precursor or inhibitor. The luciferase activities were then analyzed $24 \mathrm{~h}$ later. As shown in Fig. 6A, miR-10b precursor remarkably suppressed the activity of RLuc containing the seed sequences of E-cadherin, Apaf-1 and PTEN compared with the empty vector group. In contrast, miR-10b inhibitor greatly upregulates RLuc activity. As expected, when the mutant vectors were transfected, neither
miR-10b precursor nor inhibitor has apparent effects on the RLuc activity. All these data demonstrated that miR-10b specifically targets E-cadherin, Apaf-1 and PTEN.

To further confirm that miR-10b targets E-cadherin, Apaf-1 and PTEN, U251 cells were transfected with miR-10b precursor of inhibitor (Fig. 6B) and target gene mRNA and proteins levels were detected. Results showed that miR-10b precursor enforced expression of miR-10a markedly repressed, whereas miR-10b inhibitor significantly promoted E-cadherin, Apaf-1 and PTEN transcription (Fig. 6C). Finally, western blotting was used to detect the protein levels of these genes and the data were consistent with the mRNA levels (Fig. 6D). Collectively, these findings indicated that E-cadherin, Apaf-1 and PTEN are specifically regulated by miR-10b in GBM cells and TGF- $\beta 1$ regulates these molecules at least partly via repressing $\mathrm{miR}-10 \mathrm{~b}$. 

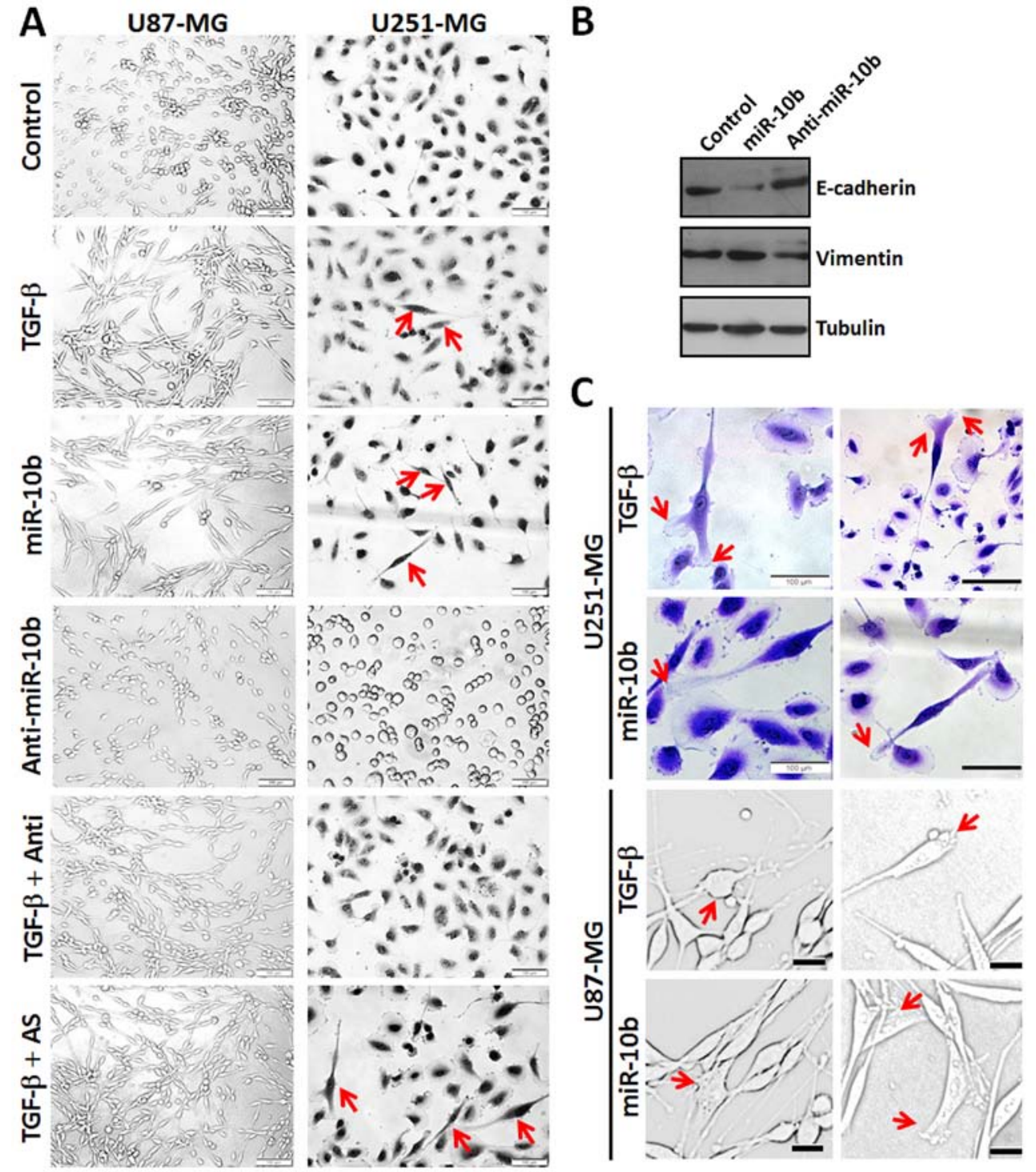

Figure 5. miR-10b promotes GBM cell EMT and lamellipodia formation. (A) U87 and U251 cells were treated as indicated and cell morphology was then observed and arrows indicate cells in fusiform shape. Bar, $100 \mu \mathrm{m}$. (B) Western blot analysis of E-cadherin and vimentin in U251 cells treated with TGF- $\beta 1$ or miR-10b for $24 \mathrm{~h}$. Tubulin was used as the internal loading control. (C) U87 and U251 cells were treated with TGF- $\beta 1$ or miR-10b and cell lamellipodia formation was observed under a microscope and arrows indicate lamellipodia. Bar, $100 \mu \mathrm{m}$ (U251) or $20 \mu \mathrm{m}$ (U87). Data are representative of three independent experiments. miR-10b, miR-10b precursor; anti-miR-10b/Anti, miR-10b inhibitor; AS, inhibitor scramble.

AntagomiR-10b enhances antitumor efficacy in glioblastoma xenografts. Our in vitro studies proved that TGF- $\beta 1$ regulates GBM cell proliferation, migration and EMT at least partially through promoting miR-10b expression. Thus, miR-10b can be used as a therapeutic target in GBM treatment. To evaluate these effects in vivo, U87 cells were stably transfected with $400 \mathrm{nM}$ agomiR-10b (mimics), antagomiR-10b (inhibitor) or agomir-control (negative control) and subcutaneous xenografts were established by subcutaneously injecting $2 \times 10^{6}$ wild-type or stably transfected U87 cells ( $\mathrm{n}=10 \mathrm{mice} / \mathrm{group}$ ). Tumor growth was under surveillance and tumor volumes were calculated with equation. As indicated in Fig. 7A, treatment with TGF- $\beta 1$ or miR-10b agomir significantly promoted GBM tumor growth, whereas the miR-10b antagomir remarkably inhibited tumor growth, even in the presence of TGF- $\beta 1$. Consistent with in vitro results, the expression of miR-10b is increased in TGF- $\beta 1$ or miR-10b agomir treated tumor tissues, and decreased in miR-10b antagomir treated tumor tissues regardless of TGF- $\beta 1$ treatment. As expected, the expression of downstream target of miR-10b is also consistent with the expression of miR-10b (Fig. 7B and C). Collectively, these data positively support our in vitro data that miR-10b acts as a key factor downstream of TGF- $\beta 1$, contributing to GBM tumor growth.

\section{Discussion}

GBM is the most common and aggressive cancer within the brain and it represents $\sim 15 \%$ of brain tumors. Although surgery, chemotherapy and radiation have been used to treat GBM patients, the high mobility and strong invasive properties of GBM result in a high inevitable recurrence rate and a poor median survival for patients $(2,32)$. The most common survival is $12-15$ months with only $<3-5 \%$ of patients surviving 

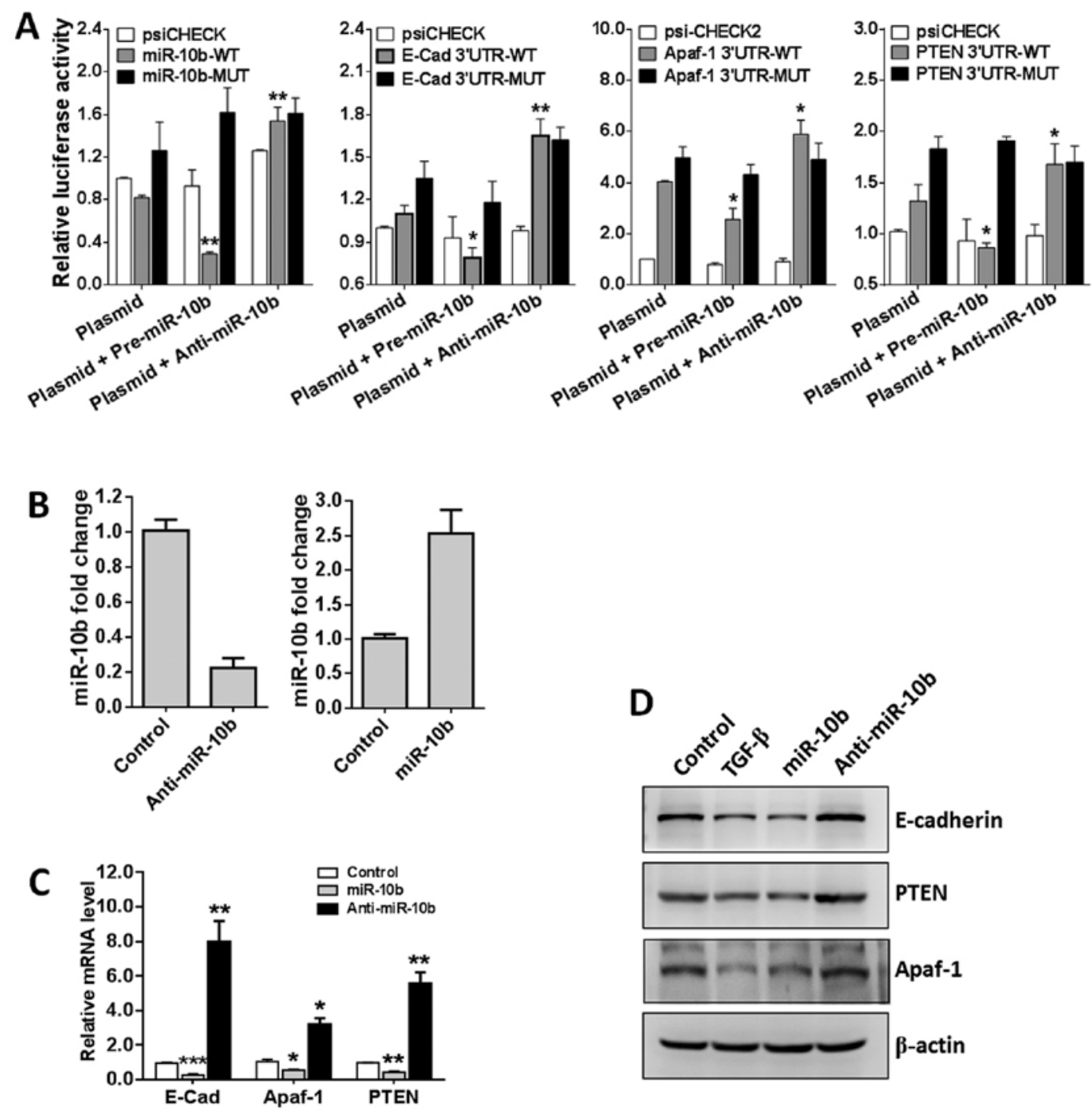

Figure 6. E-cadherin, Apaf-1 and PTEN are target genes of miR-10b. (A) Dual-luciferase reporter assays using vectors constructed with 3'-UTR of wild-type or mutant E-cadherin, Apaf-1 and PTEN /mmiR-10b-MUT alone, or in the presence of miR-10b precursor (Pre-miR-10b) or inhibitor (anti-miR-10b) were performed. Vectors constructed with miR-10b-WT or miR-10b-MUT was used as the positive control. The Renilla luciferase (RLuc) was normalized to firefly luciferase (FLuc) activity and the recombinant vector was normalized to the empty psiCHECK-2 vector. (B) The miR-10b expression levels in miR-10b mimic (miR-10b)- and miR-10b inhibitor (anti-miR-10b)-transfected U251 cells were detected by real-time PCR. The mRNA and the protein expression levels of E-cadherin, PTEN and Apaf-1 in indicated cells were detected by (C) real-time PCR and (D) western blot analysis. ${ }^{*} \mathrm{P}<0.05,{ }^{* *} \mathrm{P}<0.01,{ }^{* * *} \mathrm{P}<0.001 \mathrm{compared}$ with the control group.
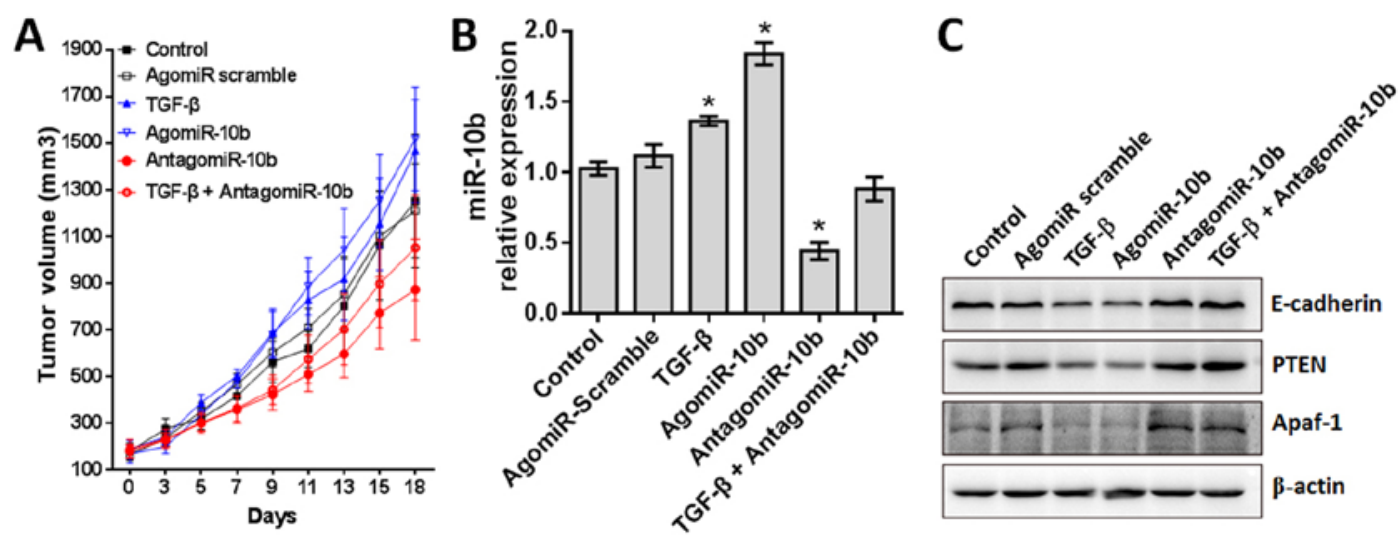

Figure 7. AntagomiR-10b reverses TGF- 31 -induced tumor growth of GBM in vivo. (A) Six groups of nu/nu nude mice with U87 xenograft were treated as indicated. Each group included 10 mice. Tumor size was measured once every 2 days. (B) The expression of miR-10b in tumor tissues from various groups was detected by real-time PCR. "P<0.05 compared with the control group. (C) Protein expression levels of E-cadherin, PTEN and Apaf-1 in tumor tissues from various groups was detected by western blot analysis. 
more than 5 years (32). Thus, there is a pressing need to reveal the molecular mechanisms of GBM proliferation and invasion for novel therapeutic avenue development.

TGF- $\beta 1$ is a multifaceted cytokine that can regulate proliferation, differentiation and other functions of various cell types including T cell (33), B cell $(34,35)$, myeloid cell and tumor cells via both Smad-dependent and Smad-independent signaling pathways $(36,37)$. TGF- $\beta 1$ plays vital roles in controlling tumor progression. On one hand, TGF- $\beta 1$ can indirectly promote tumor cell proliferation and metastasis through promoting regulatory $\mathrm{T}$ cell (Treg) differentiation. The CD4 ${ }^{+}$Foxp $3^{+}$Treg cells can hinder effective host immune responses against cancer cells and abundant Treg cell infiltration into tumors is usually associated with poor clinical outcomes $(38,39)$. On the other hand, TGF- $\beta 1$ can directly regulate tumor progression with different activities at different developmental stages $(40,41)$. However, the broad spectrum expression and functional diversity of TGF- $\beta 1$, and sometimes even coexisting with complicated tumor circumstances or other cytokines, make it difficult to clarify the roles that TGF- $\beta 1$ played in tumor development. As a result, the data on TGF- $\beta 1$ in various tumors are controversial and it has a dual role as either a tumor suppressor or promoter depending on the stages and types of the tumor $(3,4)$. Thus, further studies are still needed to elucidate the detailed mechanisms and to clarify the universal rule and essence of TGF- $\beta 1$ in GBM and other tumors.

As an oncogenic miRNA, miR-10b can regulate growth and metastasis of various types of cancer (13-15). miR-10b widely participated in the regulating of breast cancer proliferation and metastasis $(42,43)$. However, miR-10b may play diverse roles in different tumors. Accumulating evidence showed that miR-10b is increased in breast cancers (43), but only reduced expression of miR-10b was found in cervical cancer (44). The expression level of miR-10b in colorectal cancer is paradoxical as reported by different groups $(45,46)$. All these data collectively indicated that the activity of miR-10b is accurately tuned in different tumors or the same tumor at different stages. Although several target genes of miR-10b have been designated in GBM and other tumors, their regulation appears cell- and context-specific $(13,16,17)$. Thus, further studies are still needed to uncover the detailed mechanisms underlying miR-10b functions in regulating GBM progression.

Here, we investigated the role of miR-10b in TGF- $\beta 1$ mediated GBM proliferation, migration and EMT. We found that miR-10b is apparently upregulated by TGF- $\beta 1$ in U251 and U87 cells. Further studies uncovered that TGF- $\beta 1$ significantly promoted GBM cell proliferation, migration and EMT. All these effects were achieved through regulating miR-10b as miR-10b mimics promoted, whereas miR-10b inhibitor reversed, the effects of TGF- $\beta 1$ on U251 and U87 cell proliferation, migration and EMT. In addition, several proliferation-, migration- and EMT-associated genes including epithelial cadherin (E-cadherin), apoptotic protease activating factor 1 (Apaf-1) and phosphatase and tensin homolog (PTEN) are the targets of miR-10b. When xenograft models were used to investigate the miR-10b potency as therapeutic target in vivo, results showed that antagomiR directed against miR-10b remarkably suppressed tumor progression. In summary, our data collectively demonstrated that TGF- $\beta 1$ functions in GBM at least partially trough regulating miR-10b expression and our findings provide a rationale for targeting TGF- $\beta 1$ or miR-10b for the treatment of GBM.

Taken together, our data demonstrated the mechanism of TGF- $\beta 1$ and miR-10b in regulating GBM cell proliferation, migration and EMT. TGF- $\beta 1$ promotes miR-10b expression and the latter molecule then regulates GBM cell proliferation, migration and EMT through suppressing its downstream target genes E-cadherin, Apaf-1 and PTEN. Modulation of TGF- $\beta 1$ and miR-10b may effectively regulate these tumor-associated genes and both TGF- $\beta 1$ and miR-10b can be candidate targets for GBM treatment.

\section{Acknowledgements}

The present study was supported by grants from the Natural Science Fund of China (nos. 81301884, 81401883 and 81302173), the Science and Technology Department of Jilin Province (nos. 20140520036JH, 20160414052GH and 20160101053JC), the Bethune Project Plan B of Jilin University (no. 450060521279) and the First Prize of China Postdoctoral Science Foundation (no. 2016M590264).

\section{References}

1. Stupp R, Mason WP, van den Bent MJ, Weller M, Fisher B, Taphoorn MJ, Belanger K, Brandes AA, Marosi C, Bogdahn U, et $a$; : European Organisation for Research and Treatment of Cancer Brain Tumor and Radiotherapy Groups; National Cancer Institute of Canada Clinical Trials Group: Radiotherapy plus concomitant and adjuvant temozolomide for glioblastoma. N Engl J Med 352: 987-996, 2005.

2. Otsuki N, Konno T, Kurahashi T, Suzuki S, Lee J, Okada F Iuchi Y, Homma T and Fujii J: The SOD1 transgene expressed in erythroid cells alleviates fatal phenotype in congenic NZB/ NZW-F1 mice. Free Radic Res 50: 793-800, 2016.

3. Xie LP, Chen QX, Huang H, Liu XD, Chen HT and Zhang RQ: Inhibitory effects of cupferron on the monophenolase and diphenolase activity of mushroom tyrosinase. Int J Biochem Cell Biol 35: 1658-1666, 2003.

4. Lamouille S, Xu J and Derynck R: Molecular mechanisms of epithelial-mesenchymal transition. Nat Rev Mol Cell Biol 15: 178-196, 2014.

5. Brown KA, Aakre ME, Gorska AE, Price JO, Eltom SE Pietenpol JA and Moses HL: Induction by transforming growth factor-betal of epithelial to mesenchymal transition is a rare event in vitro. Breast Cancer Res 6: R215-R231, 2004.

6. Chen $\mathrm{K}$ and Rajewsky N: The evolution of gene regulation by transcription factors and microRNAs. Nat Rev Genet 8: 93-103, 2007.

7. Wei F, Liu Y, Guo Y, Xiang A, Wang G, Xue X and Lu Z: miR-99b-targeted mTOR induction contributes to irradiation resistance in pancreatic cancer. Mol Cancer 12: 81, 2013.

8. Mu N, Gu J, Huang T, Zhang C, Shu Z, Li M, Hao Q, Li W, Zhang W, Zhao J, et al: A novel NF-кB/YY1/microRNA-10a regulatory circuit in fibroblast-like synoviocytes regulates inflammation in rheumatoid arthritis. Sci Rep 6: 20059, 2016.

9. Wu W, He C, Liu C, Cao AT, Xue X, Evans-Marin HL, Sun M Fang L, Yao S, Pinchuk IV, et al: miR-10a inhibits dendritic cell activation and Th1/Th17 cell immune responses in IBD. Gut 64: 1755-1764, 2015.

10. Nikaki A, Piperi C and Papavassiliou AG: Role of microRNAs in gliomagenesis: Targeting miRNAs in glioblastoma multiforme therapy. Expert Opin Investig Drugs 21: 1475-1488, 2012.

11. Costa PM, Cardoso AL, Mano M and de Lima MC: MicroRNAs in glioblastoma: Role in pathogenesis and opportunities for targeted therapies. CNS Neurol Disord Drug Targets 14: 222-238, 2015.

12. Yang W, Yu H, Shen Y, Liu Y, Yang Z and Sun T: MiR-146b-5p overexpression attenuates stemness and radioresistance of glioma stem cells by targeting HuR/lincRNA-p21/ $\beta$-catenin pathway. Oncotarget 7: 41505-41526, 2016. 
13. Ma L, Teruya-Feldstein J and Weinberg RA: Tumour invasion and metastasis initiated by microRNA-10b in breast cancer. Nature 449: 682-688, 2007.

14. Nakata K, Ohuchida K, Mizumoto K, Kayashima T, Ikenaga N, Sakai H, Lin C, Fujita H, Otsuka T, Aishima S, et al: MicroRNA-10b is overexpressed in pancreatic cancer, promotes its invasiveness, and correlates with a poor prognosis. Surgery 150: 916-922, 2011.

15. Mussnich P, D'Angelo D, Leone V, Croce CM and Fusco A: The High Mobility Group A proteins contribute to thyroid cell transformation by regulating miR-603 and miR-10b expression. Mol Oncol 7: 531-542, 2013.

16. Gabriely G, Yi M, Narayan RS, Niers JM, Wurdinger T, Imitola J, Ligon KL, Kesari S, Esau C, Stephens RM, et al: Human glioma growth is controlled by microRNA-10b. Cancer Res 71: 3563-3572, 2011.

17. Ma L, Reinhardt F, Pan E, Soutschek J, Bhat B, Marcusson EG, Teruya-Feldstein J, Bell GW and Weinberg RA: Therapeutic silencing of miR-10b inhibits metastasis in a mouse mammary tumor model. Nat Biotechnol 28: 341-347, 2010.

18. Teplyuk NM, Uhlmann EJ, Wong AH, Karmali P, Basu M, Gabriely G, Jain A, Wang Y, Chiocca EA, Stephens R, et al: MicroRNA-10b inhibition reduces E2F1-mediated transcription and miR-15/16 activity in glioblastoma. Oncotarget 6: 3770-3783, 2015.

19. Teplyuk NM, Uhlmann EJ, Gabriely G, Volfovsky N, Wang Y Teng J, Karmali P, Marcusson E, Peter M, Mohan A, et al: Therapeutic potential of targeting microRNA-10b in established intracranial glioblastoma: First steps toward the clinic. EMBO Mol Med 8: 268-287, 2016.

20. Gabriely G, Teplyuk NM and Krichevsky AM: Context effect: microRNA-10b in cancer cell proliferation, spread and death. Autophagy 7: 1384-1386, 2011.

21. Zhang M, Kleber S, Röhrich M, Timke C, Han N, Tuettenberg J, Martin-Villalba A, Debus J, Peschke P, Wirkner U, et al: Blockade of TGF- $\beta$ signaling by the TGF $\beta$ R-I kinase inhibitor LY2109761 enhances radiation response and prolongs survival in glioblastoma. Cancer Res 71: 7155-7167, 2011.

22. Joseph JV, Conroy S, Tomar T, Eggens-Meijer E, Bhat K, Copray S, Walenkamp AM, Boddeke E, Balasubramanyian V, Wagemakers M, et al: TGF- $\beta$ is an inducer of ZEB1-dependent mesenchymal transdifferentiation in glioblastoma that is associated with tumor invasion. Cell Death Dis 5: e1443, 2014.

23. Duan R, Han L, Wang Q, Wei J, Chen L, Zhang J, Kang C and Wang L: HOXA13 is a potential GBM diagnostic marker and promotes glioma invasion by activating the Wnt and TGF- $\beta$ pathways. Oncotarget 6: 27778-27793, 2015.

24. Lv S, Qin J, Yi R, Coreman M, Shi R, Kang H and Yao C: CrkL efficiently mediates cell proliferation, migration, and invasion induced by TGF- $\beta$ pathway in glioblastoma. J Mol Neurosci 51: 1046-1051, 2013.

25. Gregory PA, Bert AG, Paterson EL, Barry SC, Tsykin A, Farshid G, Vadas MA, Khew-Goodall Y and Goodall GJ: The miR-200 family and miR-205 regulate epithelial to mesenchymal transition by targeting ZEB1 and SIP1. Nat Cell Biol 10: 593-601, 2008.

26. Xiong M, Jiang L, Zhou Y, Qiu W, Fang L, Tan R, Wen $P$ and Yang J: The miR-200 family regulates TGF- $\beta 1$-induced renal tubular epithelial to mesenchymal transition through Smad pathway by targeting ZEB1 and ZEB2 expression. Am J Physiol Renal Physiol 302: F369-F379, 2012.

27. Hardee ME, Marciscano AE, Medina-Ramirez CM, Zagzag D, Narayana A, Lonning SM and Barcellos-Hoff MH: Resistance of glioblastoma-initiating cells to radiation mediated by the tumor microenvironment can be abolished by inhibiting transforming growth factor- $\beta$. Cancer Res 72: 4119-4129, 2012.

28. Joseph JV, Balasubramaniyan V, Walenkamp A and Kruyt FAE: TGF- $\beta$ as a therapeutic target in high grade gliomas - promises and challenges. Biochem Pharmacol 85: 478-485, 2013.
29. Batlle E, Sancho E, Francí C, Domínguez D, Monfar M, Baulida J and García De Herreros A: The transcription factor snail is a repressor of E-cadherin gene expression in epithelial tumour cells. Nat Cell Biol 2: 84-89, 2000.

30. Soengas MS, Alarcón RM, Yoshida H, Giaccia AJ, Hakem R, Mak TW and Lowe SW: Apaf-1 and caspase-9 in p53-dependent apoptosis and tumor inhibition. Science 284: 156-159, 1999.

31. Fang M, Zhong XY, Du B, Lin CL, Luo F, Tang LJ and Chen J: Role of DJ-1-induced PTEN down-regulation in migration and invasion of human glioma cells. Chin J Cancer 29: 988-994, 2010.

32. Gallego O: Nonsurgical treatment of recurrent glioblastoma. Curr Oncol 22: e273-e281, 2015.

33. Wahl SM, Hunt DA, Wong HL, Dougherty S, McCartneyFrancis N, Wahl LM, Ellingsworth L, Schmidt JA, Hall G, Roberts $\mathrm{AB}$, et al: Transforming growth factor-beta is a potent immunosuppressive agent that inhibits IL-1-dependent lymphocyte proliferation. J Immunol 140: 3026-3032, 1988.

34. Letterio JJ and Roberts AB: Regulation of immune responses by TGF-beta. Annu Rev Immunol 16: 137-161, 1998.

35. Kehrl JH, Thevenin C, Rieckmann P and Fauci AS: Transforming growth factor-beta suppresses human B lymphocyte Ig production by inhibiting synthesis and the switch from the membrane form to the secreted form of Ig mRNA. J Immunol 146: 4016-4023, 1991.

36. Li MO, Wan YY, Sanjabi S, Robertson AK and Flavell RA: Transforming growth factor-beta regulation of immune responses. Annu Rev Immunol 24: 99-146, 2006.

37. Qin H, Wang L, Feng T, Elson CO, Niyongere SA, Lee SJ, Reynolds SL, Weaver CT, Roarty K, Serra R, et al: TGF-beta promotes Th17 cell development through inhibition of SOCS3. J Immunol 183: 97-105, 2009.

38. Curiel TJ, Coukos G, Zou L, Alvarez X, Cheng P, Mottram P, Evdemon-Hogan M, Conejo-Garcia JR, Zhang L, Burow M, et al: Specific recruitment of regulatory $\mathrm{T}$ cells in ovarian carcinoma fosters immune privilege and predicts reduced survival. Nat Med 10: $942-949,2004$

39. deLeeuw RJ, Kost SE, Kakal JA and Nelson BH: The prognostic value of FoxP3 ${ }^{+}$tumor-infiltrating lymphocytes in cancer: A critical review of the literature. Clin Cancer Res 18: 3022-3029, 2012.

40. Jiang Y, Woosley AN, Sivalingam N, Natarajan S and Howe PH: Cathepsin-B-mediated cleavage of disabled- 2 regulates TGF- $\beta$ induced autophagy. Nat Cell Biol 18: 851-863, 2016.

41. Akhurst RJ and Derynck R: TGF-beta signaling in cancer - a double-edged sword. Trends Cell Biol 11: S44-S51, 2001.

42. Knirsh R, Ben-Dror I, Modai S, Shomron N and Vardimon L: MicroRNA 10b promotes abnormal expression of the protooncogene c-Jun in metastatic breast cancer cells. Oncotarget 7: 59932-59944, 2016.

43. Bahena-Ocampo I, Espinosa M, Ceballos-Cancino G, Lizarraga F, Campos-Arroyo D, Schwarz A, Garcia-Lopez P, Maldonado V and Melendez-Zajgla J: miR-10b expression in breast cancer stem cells supports self-renewal through negative PTEN regulation and sustained AKT activation. EMBO Rep 17: 1081,2016

44. Zou D, Zhou Q, Wang D, Guan L, Yuan L and Li S: The Downregulation of MicroRNA-10b and its role in cervical cancer. Oncol Res 24: 99-108, 2016.

45. Vychytilova-Faltejskova P, Pesta M, Radova L, Liska V, Daum O, Kala Z, Svoboda M, Kiss I and Slaby O: Genome-wide microRNA expression profiling in primary tumors and matched liver metastasis of patients with colorectal cancer. Cancer Genomics Proteomics 13: 311-316, 2016.

46. Wang Y, Li Z, Zhao X, Zuo X and Peng Z: miR-10b promotes invasion by targeting HOXD10 in colorectal cancer. Oncol Lett 12: 488-494, 2016. 